\title{
Electro-mechanical coupled design of self-powered sensing systems and performances comparison through experiments
}

\author{
A. Somà, G. De Pasquale \\ Department of Mechanical and Aerospace Engineering, Politecnico di Torino \\ Corso Duca degli Abruzzi 24, 10129 Torino (Italy) \\ aurelio.soma@polito.it,giorgio.depasquale@polito.it
}

\begin{abstract}
Recent advances in low-power sensors and electronic components open to innovative strategies in structural monitoring and real-time data processing, in particular for industrial and vehicular fields. Dedicated devices for harvesting the energy dissipated by mechanical vibrations of machines are showing their applicability in supplying autonomous distributed sensing systems. The harvester will replace cables and storage batteries, with relevant benefits on the sensing system capillarity, accessibility and applicability. The design of the interfaces of the electric, magnetic and structural coupled systems forming the harvester include static and dynamic modeling and simulation of the interactions involved; smart and effective architectures are need to satisfy the general requirements of bandwidth, tunability and efficiency required by each application. This paper reports the research advances in this field as a result of laboratory tests and design studies, with particular focus on the design methodologies involved in the definition of energy harvesters.
\end{abstract}

KEYwORDS. Energy harvesting; Structural monitoring; Autonomous sensing; Bandwidth; Resonance tuning; Duty cycle; Efficiency.

\section{INTRODUCTION}

$\mathrm{T}$ here are many unexploited energy sources in the environment such as light, wind, temperature gradients, radiofrequency waves, kinetic energy of sea waves, mechanical vibrations, human body motion, etc. The conversion of the unused energy in electricity, called 'energy harvesting', is motivating many academic and industrial researches in the last years [1-3]. Due to the small power amount that is usually available, the energy harvesting is mainly addressed to the supply of low consumption technologies. The harvesting of energy from the environment represents a valid alternative to batteries and cables, and promises to open new chances of development for mobile and wireless devices. Energy harvesters will allow using, for example, wireless sensors in many applications such as industrial and structural monitoring, remote medical assistance, military equipments, materials flows monitoring, transports and logistics, energetic efficiency control. Without the capabilities of energy harvesting devices, it is almost impossible to supply sensing devices in critical positions where accessibility is limited, for example in telemedicine applications and biological parameters monitoring. Miniaturized and wearable harvesters allow continuous monitoring of patients affected by chronic pathologies by means of sensors integrated in the body that do not need batteries for the supply of energy.

This study compares some different strategies for harvesting energy from the environment, with particular attention to mechanical vibrations. In previous works, the authors analyzed the performances of different energy harvesters: piezoelectric [4], magnetic inductive [5], electrostatic capacitive, and magnetically levitated [6]. Also they patented some dedicated devices [7-9]. In this paper, the results of simulation and testing of different energy harvesters are introduced and compared in terms of energy generated per unit volume. Then, some design solutions for the integration of the harvester in monitoring devices for vehicles and mechanical systems are analyzed. Finally, after the comparison of 
different conversion strategies, two typologies of harvesters are characterized for obtaining design indications about the autonomous sensing system.

\section{VIBRATING ENERGY CONVERSION STRATEGIES}

7 he kinetic energy associated to environmental vibrations is generally exploited by harvesters to excite the oscillation of a seismic inertial mass connected to the transducer. The motion induced on the seismic mass is regulated in amplitude, frequency and phase angle in order to maximize the conversion efficiency. The most diffuse conversion principles are piezoelectric, magnetic-inductive and capacitive (Tab. 1). In the following, the most important characteristics of these three conversion strategies are discussed.

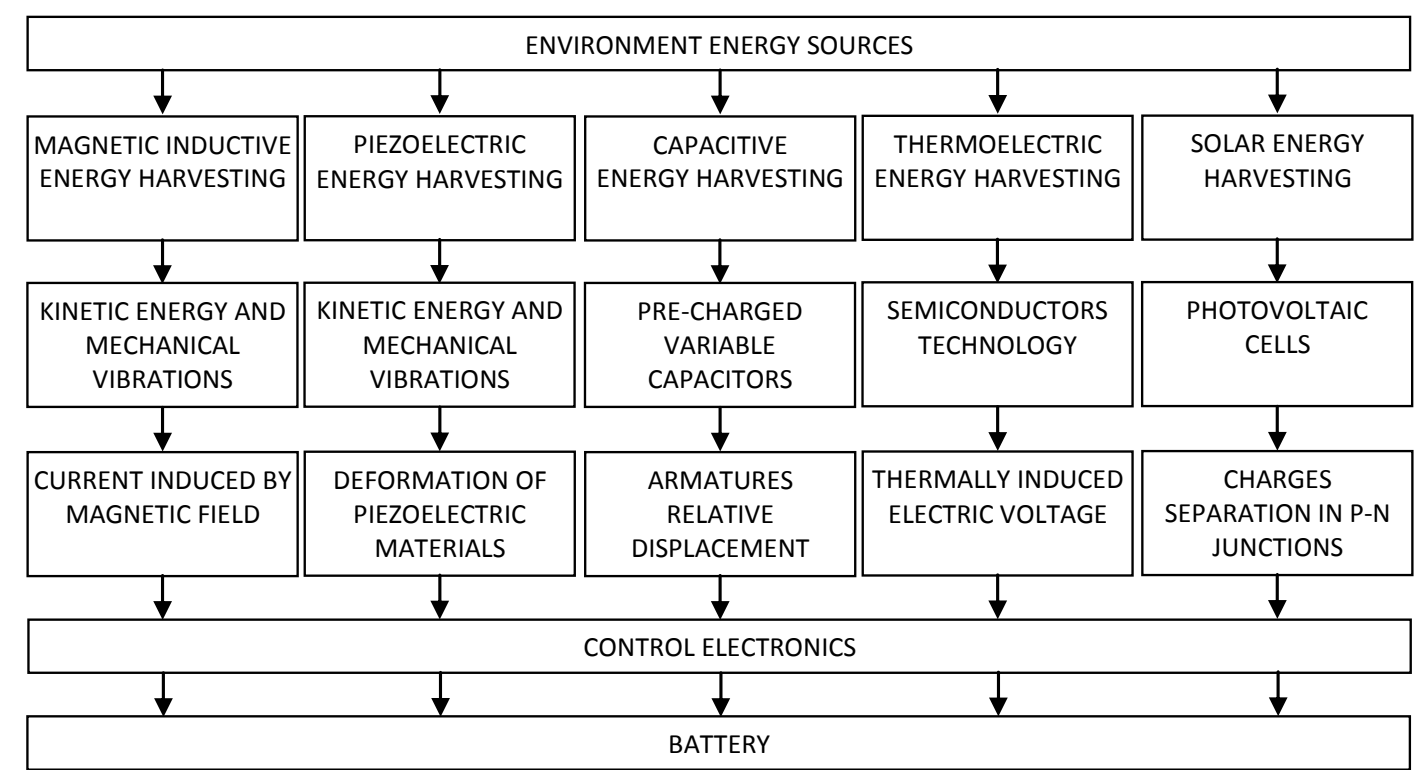

Table 1: Some typologies of energy harvesters.

\section{Piezoelectric energy harvesters}

In this typology of generators, the seismic mass induces the deformation of a piezoelectric component that produces a voltage difference between its electrodes proportional to the mechanical strain. About piezo generators, some reliability limitations have been pointed out in the literature because of the brittleness of materials used (PZT, PVDF, etc). The electric output power is function of the resistive load applied to the transducer; then, the preliminary characterization of the impedance of the electronic conditioning circuit is needed. The shape of the output signal under sinusoidal excitation represents another possible source of inefficiency; in fact, current and voltage waves are slightly phase shifted due to the intrinsic capacitance of the piezoelectric material. This reduces the effective output power with respect to the optimized output power theoretically available in case of preliminary phase synchronization of current and voltage; unfortunately this operation needs relevant complications of the conditioning circuit and is generally omitted. Usually, the output voltage is rather high (in the order of tens of $\mathrm{V}$ ), while the current is generally low (in the order of $\mathrm{mA}$ ); when the storage battery is included in the autonomous sensing system, the voltage/current ratio should be modified in order to reduce the time of charge. This operation also requires additional circuit complications.

The advantages related to piezoelectric generators are given by the large commercial availability of transducers, which are almost ready for the integration in the harvesting system. Furthermore, they are characterized by good ratios of generated power per unit volume. From the dynamic viewpoint, these typology of generators can be applied to moderately wide ranges of frequencies (up to few hundreds of $\mathrm{Hz}$ ), which are typically associated to structural vibrations. The resonance tuning can be achieved by varying the stiffness of the deformable element or by changing the seismic mass. Instead, the active tuning able to set the resonance on the actual working regime and bandwidth is generally more complicated. An example of piezoelectric generator is reported in Fig. 1. 


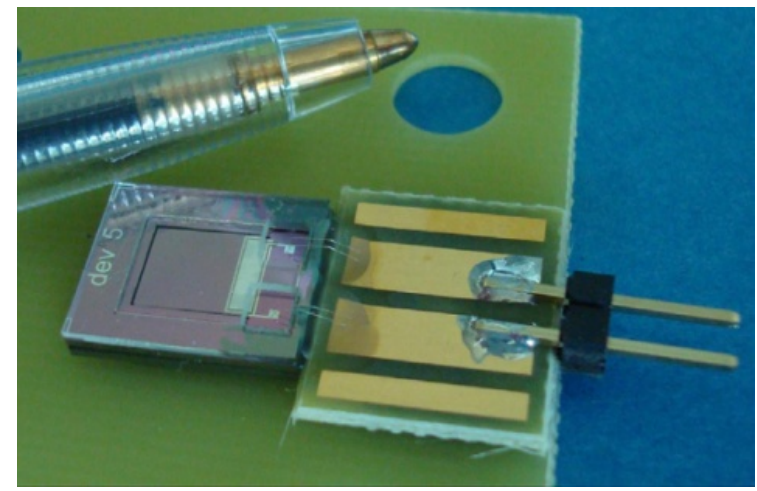

Figure 1: Piezoelectric generator [10]: 10x10x1 $\mathrm{mm}^{3}$ size, $60 \mu \mathrm{W}$ output power in resonance condition $(572 \mathrm{~Hz})$ and $2 \mathrm{~g}$ acceleration.

\section{Magnetic inductive energy harvesters}

In this case, the vibrating energy is used to induce the relative motion between a permanent magnet and a conductive coil. In the literature, a number of design solutions have been introduced, which include fixed magnet and movable coil or, more frequently, fixed coil and movable magnet. The design activity involves the selection of the coil diameter and turns number, which are both related to the current produced and to the electric resistance of the wire. By varying these two parameters, the optimized solution can be found in terms of conversion efficiency. Other important design parameters are the magnetic material and the magnet dimensions. Generally, magnets characterized by high induced magnetic field are preferable, as the so-called 'rare earths' magnets (e.g. NdFeB). The most important benefits in using inductive harvesters are the stability of performances, the high reliability and, above all, the high current/voltage ratio that makes these generators suitable for charging batteries without complicated electronic conditioning. Unfortunately, the integration of tuning controls is generally hard because the resonance of the harvester is dominated by the mass properties of the magnet, which is already dimensioned to fit the electric requirements. Furthermore, the power generation is strongly linked to the magnet velocity, with reference to the relation between the current induced in the coil and the gradient of the magnetic field expressed by the Faraday law. This indicates that high output power is obtainable from long travels of the magnet and, consequently, by dedicated design of suspensions. The lowering of suspension stiffness can be obtained, for instance, by using magnetic springs represented by repulsing magnets. Fig. 2 reports an example of inductive energy harvester from the literature.

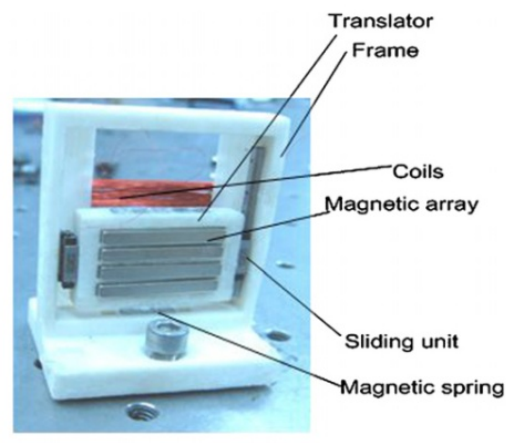

Figure 2: Magnetic-inductive energy harvester [11]: $54 \times 46 \times 15 \mathrm{~mm}^{3}$ size, $0.55 \mathrm{~mW}$ output power at $9.25 \mathrm{~Hz}$ frequency and $0.8 \mathrm{~g}$ acceleration.

\section{Capacitive energy harvesters}

Capacitive generators are basically capacitors with movable armatures where the relative displacement of the armatures is induced by external vibration and is used to generate voltage or charge difference between them. The electric power generated is strongly dependent to the capacitor geometry: in particular, small gaps between armatures and large armature surfaces are particularly advantageous. However, the pre-charge of armatures is needed to generate energy and it represents serious limitation to the efficiency of the energy harvester. In order to reduce as much as possible the charging energy, very small armatures surfaces are preferred. Then, in practice, the application of capacitive energy harvesters is limited to small scales and some examples are available in the field of MEMS (micro electro-mechanical systems). The 
mentioned properties represent the most relevant drawbacks of capacitive generators; another limitation is given by the high dynamic response of the harvester that is imposed by the small masses used, which reduces the applicability of these devices to high frequencies (in the order of $\mathrm{kHz}$ ).

However, in the microscale, the technology required to fabricate this typology of devices (i.e. surface micromachining) is consolidated and good constructive solutions are achievable. Recently, it was documented the possibility to apply particular materials (electrets) on the variable capacitor surfaces; these materials have intrinsic charge and they are able to preserve this charge for long time compared to the harvester life. This technological improvement makes unnecessary the electric preload and sensitively increases the device efficiency. Fig. 3 reports an example to MEMS capacitive harvesters and a harvester with electret.

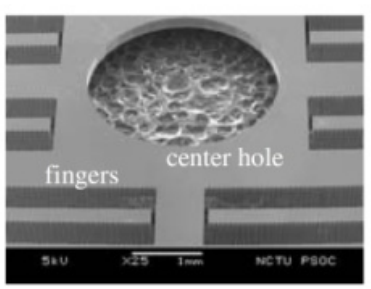

(a)

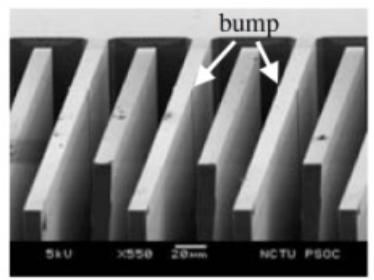

(c)

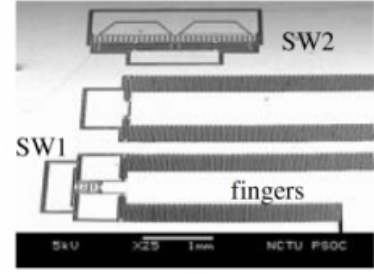

(b)

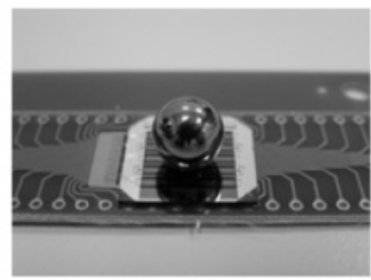

$(d)$

(a)

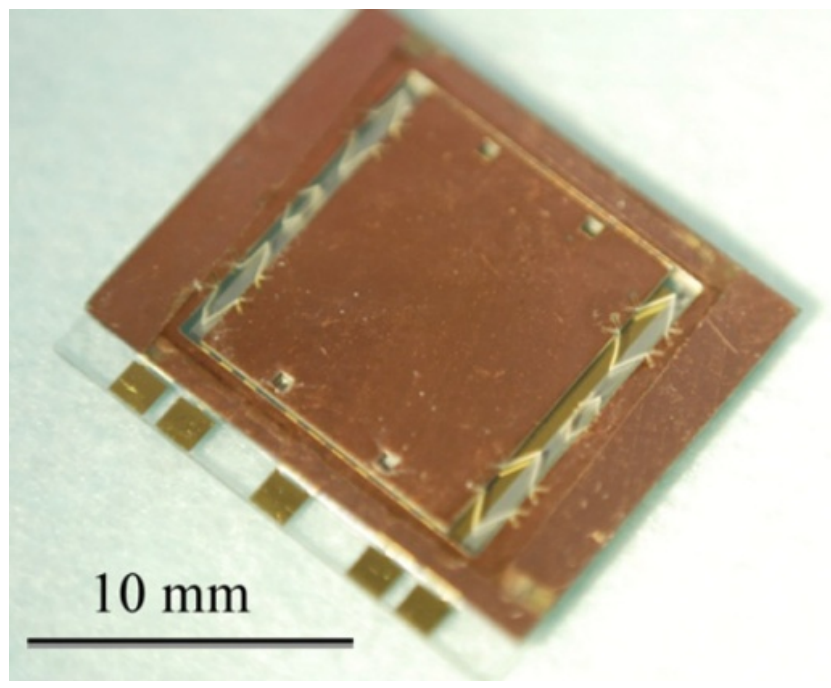

(b)

Figure 3: (a) Capacitive generator [12] including the seismic mass represented by a tungsten ball (4mm diameter) for the frequency

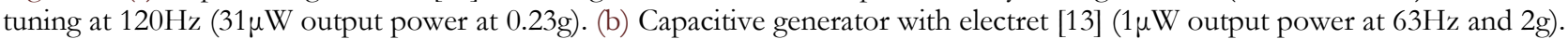

\section{POWER SPECTRAL DENSITY, FREQUENCY TUNING AND BANDWIDTH AMPLIFICATION}

$\mathrm{F}$ rom the methodological viewpoint, the preliminary analysis of the vibrating excitation source is needed before to dimension the harvester. In general applications, the input force has random shape because it is produced by machines or mechanical systems having variable operating regimes (e.g., vehicles in motion at different velocities or aircrafts in different flight conditions). The average energy associated to the input signal can be evaluated, for instance, from the acceleration associated to every specific working condition or, more generally, from the overall acceleration range. After estimating the available energy, then is possible to define, at least roughly, the size of the harvester and the size of the oscillating parts. The goal of this first design tentative is to provide the order of magnitude of masses and suspensions stiffness with reference to the response/excitation amplitude ratio. In other words, the dynamic parameters of the generator are defined in dependence to the desired FRF.

Next step addresses to the analysis of energy distribution in the frequency spectrum. Usually, the environmental oscillation is amplified in correspondence to particular frequencies because of multiple resonances and combined modal couplings that interest the machine or mechanical system hosting the harvester. In correspondence to the machine resonance, the available energy coming from the vibrating environment is amplified. The description of the energy distribution in the frequency domain is provided by the PSD (power spectral density) function that must be considered accurately during the next part of the dimensioning.

The harvester tuning consists in modifying the mass and stiffness parameters that have been approximately defined in the very preliminary dimensioning. The dynamic parameters are finalized in detail at this stage; generally, their values are constant for the overall generator life: this is the simpler approach that well fits environments characterized by regular vibrations and repeatable PDS. However sometimes the working regimes are strongly variable and the energy amount associated to vibrations is very scarce; in these cases, the adoption of active tuning systems able to change the dynamic 
response of the generator is needed. Unfortunately, active tuning systems generally cause additional power consumption and intense optimization activities are required to preserve acceptable values of efficiency. Tuning systems with fixed response are, for example, arrays of vibrating structures with slightly variable geometry, stiffness variation by preloading, additional masses, etc. Tuning systems providing variable response to the harvester are, for example, movable constraints, movable masses, variable external forces (e.g. magnetically induced), etc.
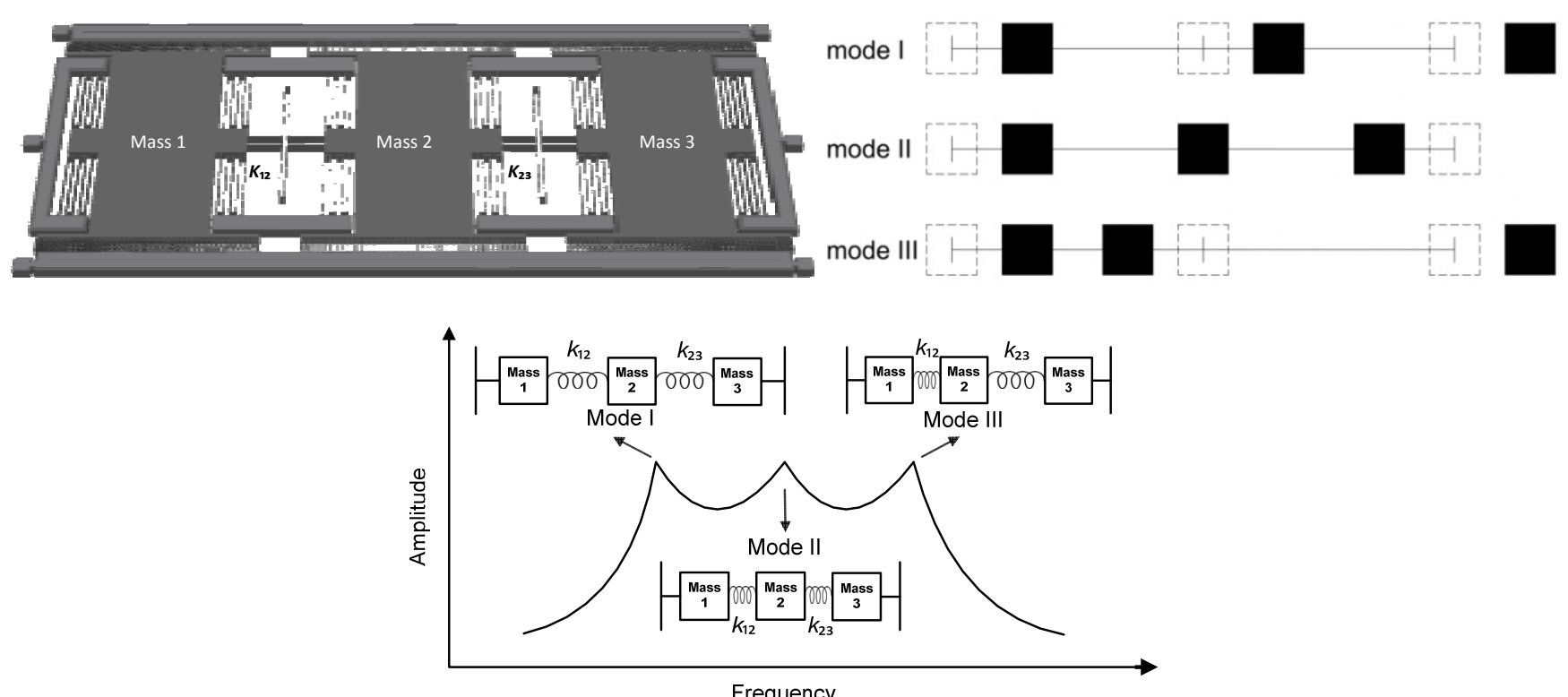

Figure 4: Example of design strategy for amplifying the bandwidth of a capacitive generator through the coupling of modal shapes.

Another important parameter for the generator is the bandwidth. From the dynamic viewpoint, vibration harvesters behave like mechanical filters: the excitation signal induces the movement of the oscillating system according to its dynamic behavior. The harvester response is directly proportional to the generated energy, because the dynamic response drives the electro-mechanical transducer, independently to the typology. Obviously, wide band generators are more performing than narrow band generators because the first ones are able to catch the energy associated to external forces in larger frequency ranges. In case of high variability of the excitation force, corresponding to very distributed PSD, wide band harvesters are more suitable. There are many strategies to amplify the bandwidth; the most diffused are the modal coupling of many transducers (Fig. 4), the series coupling of multiple generators, and the use of bi-stable structures.

\section{SYSTEM ARCHITECTURE AND DUTY CYCLE}

$\mathrm{T}$ he activity of design and dimensioning of the generator must be supported by the detailed information about the characteristics of the overall autonomous system including its performances, sampling rate, frequency of data transmission, etc. For this reason, the best definition of this step of activity is 'self-powered system design' instead of simply 'energy harvester design'; in fact, the definition includes also the electrical parameters of the utilizer and the device performances. Although the typology of components included in the autonomous system may vary among the applications, usually the following functional blocks can be identified (Fig. 5): current rectifier, charge reservoir (storage battery), one or more sensing devices (sensors), and transceiver device. Very frequently the energy generated by the harvester is stored in the battery before to be used; this operation requires the preliminary conversion of the alternate current produced by the harvester in continuous current. The charge storing, is an expensive activity in terms of energetic efficiency, however it is often necessary due to some reasons: firstly, to provide continuous supply also in case of irregular power supply; secondly, to reach the prescribed energy threshold needed to supply the utilizer.

The energy produced by the harvester and stored in the battery can be used for the supplying only when the minimum charge threshold is reached; this lower level of the battery charge is imposed by the energetic demand of the utilizers. Similarly, the time interval when the power flows from the battery to the utilizer must be calculated in function to the 
energy consumed in the unit time. The constraints mentioned are well considered in defining the duty cycle of the selfpowered system. The adoption of dedicated strategies addressed to the reduction of the energy consumption is vital for the proper working of the system; for instance, power management operations should be implemented, which consists in activating the system only above specified charge threshold of the battery (triggering) or in switching-off some components (e.g. the antenna) during data measurement and processing.

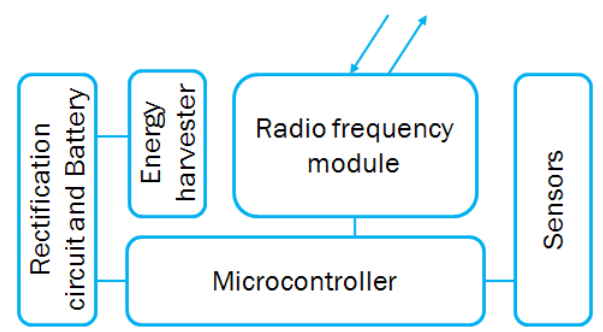

Figure 5: Typical architecture of self-powered sensing system.

\section{EXPERIMENTAL COMPARISON OF PERFORMANCES}

$\mathrm{T}$ his section reports the experimental characterization of the performances of two typologies of energy harvesters: piezoelectric and magnetic inductive. The generators are tuned during the tests by modifying their resonance through additional masses or by changing the stiffness of the deformable parts. Different values of the input acceleration are imposed, in order to verify the variation of the electro-mechanical response. The measurements were conducted by using the dedicated test equipments described in the following.

\section{Test bench}

The test bench for the experimental tests is composed by the parts reported in the following scheme (Fig. 6).

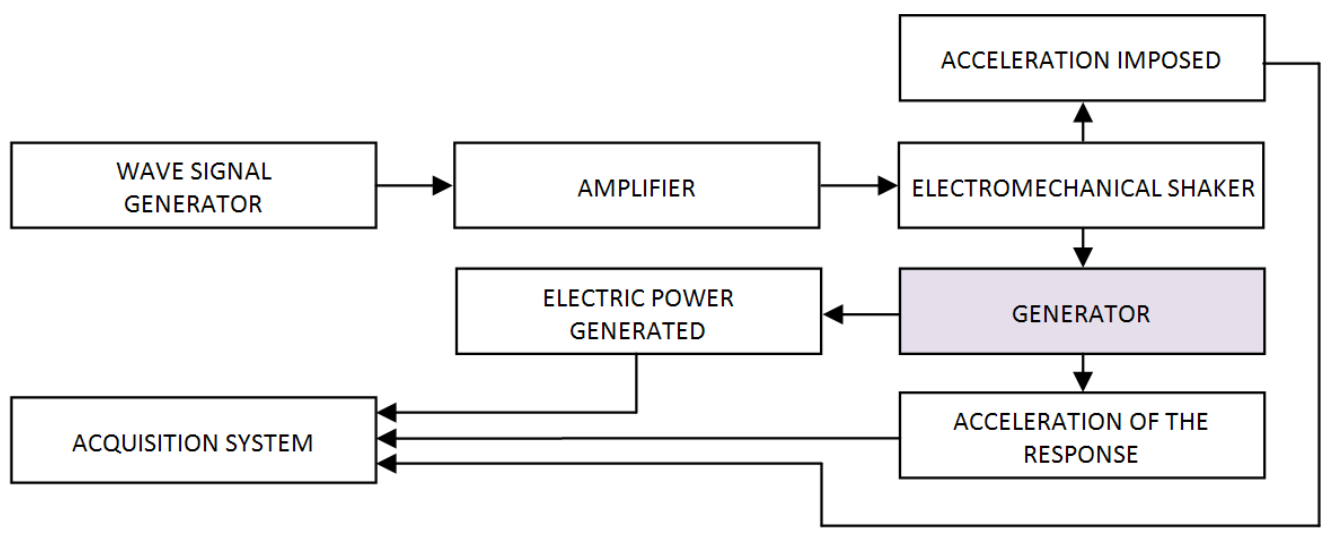

Figure 6: Blocks diagram of the test bench for the experimental characterization of the harvesters.

\section{Piezoelectric generators}

Two types of piezoelectric generators are reported in Fig. 7. The first generator is commercialized by Cedrat Technologies for applications in the aeronautic field [14], the second generator is a laboratory prototype fabricated for harvesting energy from railway vehicles and integrates the transducer DuraAct P-876.A12 [4]. Their principal characteristics are summarized in Tab. 2.

The commercial harvester is composed by two piezoelectric blocks situated within a metallic frame that, under vibrations applies the tensile-compressive load to the electro-mechanical transducer according to modal deformation. The selected design gives high stiffness to the structure $(100 \mathrm{~N} / \mathrm{mm}$ nominal) and increases the resonance frequency up to about 400 $\mathrm{Hz}$. The laboratory prototype, instead, is based on the deformation of the piezoelectric cantilever in the flexural mode: this configuration allows maximizing the ratio between the material strain and the applied force. Then, the harvester stiffness can be reduced to only $0.06 \mathrm{~N} / \mathrm{mm}$ and the resonance frequency is lowered to about $27 \mathrm{~Hz}$. The drawbacks of 
the second solution are mainly related to the reduced reliability of the transducer, which is made of brittle material subjected to fatigue effect. However, the polymeric package that coats the piezo surface of the commercial transducer provides sensitive improvements to the component lifetime (longer than $10^{6}$ cycles with $1 \mathrm{~g}$ acceleration).

\begin{tabular}{ccc}
\hline & Cedrat generator & Laboratory prototype \\
\hline Dimensions $(\mathrm{mm})$ & $48 \times 13 \times 10$ & $60 \times 35 \times 0.5$ \\
Capacity $(\mathrm{nF})$ & 3150 & $90-150$ \\
Optimized load $(\mathrm{k} \Omega)$ & 0.47 & 200 \\
Resonance frequency $(\mathrm{Hz})$ & 405 & 27 \\
\hline
\end{tabular}

Table 2: Some properties of the two piezoelectric generators.

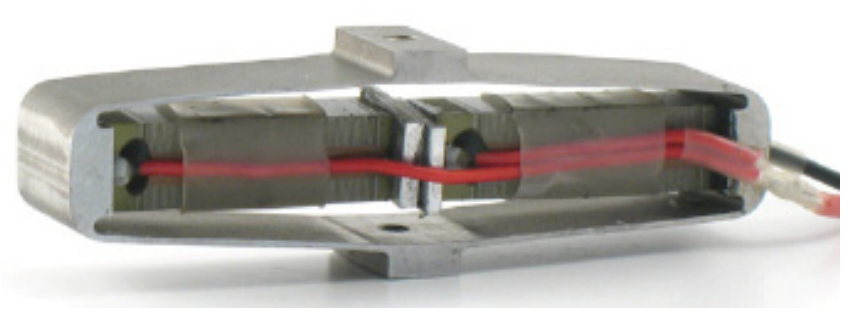

(a)

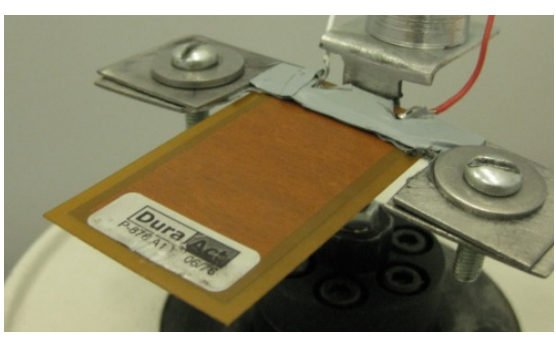

(b)

Figure 7: (a) Piezoelectric generator working in tension-compression VEH-APA 400M-MD (Cedrat Technologies); (b) laboratory prototype of flexural generator made with DuraAct P-876.A12 transducer.

The preliminary characterization of the generator, which is mandatory for piezoelectric harvesters, is addressed to the identification of the optimum resistive load associated to the transducer. For this type of generators, the output power is function of the electric resistance connected in series to the generator. The electric resistance is partially provided by the conditioning electronics, the rectification circuit and other electronic components. The characterization revealed that the commercial transducer has the optimum load at $0.47 \mathrm{k} \Omega$ and the laboratory prototype at $200 \mathrm{k} \Omega$.

Fig. 8 reports the power curves referred to the generators. Different masses were applied to the metallic frame in the first case and to the cantilever tip in the second case to modify the harvester response. In Fig. 8a it is clearly visible the shift of the resonance peak due to the mass change; this strategy is suitable for tuning the resonance of the generator on different values of the excitation frequency. In Fig. 8b, the ratio seismic mass/deformation is sensitive to the transducer configuration for constant input acceleration. In this case, all measurements are referred to the system resonance.

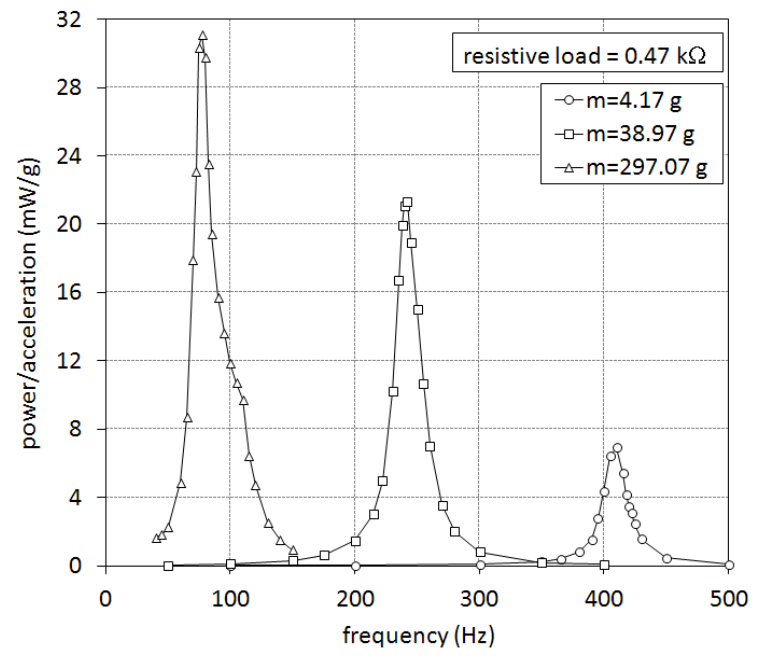

(a)

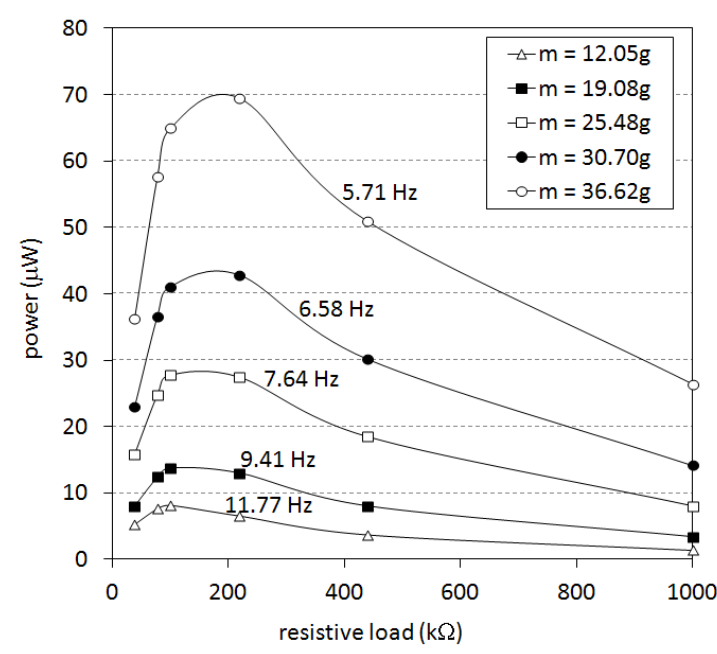

(b)

Figure 8: Output power generated by commercial harvester as function of the excitation frequency (a) and by laboratory prototype as function of the resistive load in resonance conditions $(27 \mathrm{~Hz})$ for variable seismic mass $(\mathrm{b})$. 


\section{Magnetic inductive generators}

The schematic drawing of the magnetic inductive energy harvester designed and built by the authors in [5] is represented in Fig. 9. For this type of generators, the selection of materials and dimensions of components is crucial for the electric output power and for the system frequency tuning. The magnetic suspension representing the stiffness of the transducer is determined by the magnetic field intensity associated to the magnets with opposite polarity; similarly, the electric current induced in the coil is proportional to the size of the oscillating magnet and to its relative velocity, which are both dependent to the suspension stiffness. In conclusion, the dimensioning is significantly complicated by the strong electromechanical coupling among the components and by the variability of frequency and acceleration of the excitation resulting from its spectrum.

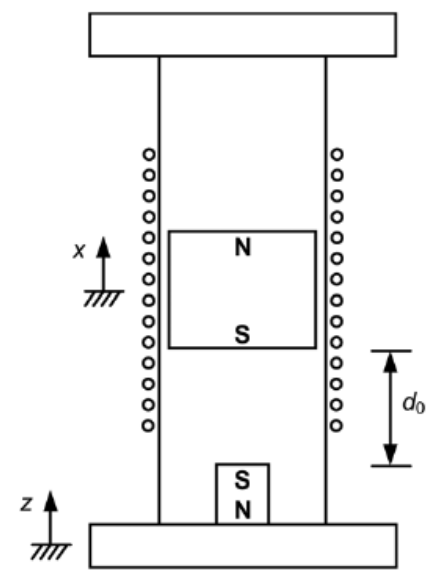

Figure 9: Schematic drawing of the magnetic inductive harvester prototype [5].

Analytical models and numerical models based on finite element method were used to calculate the force-displacement characteristic of the magnetic suspension. The goal of experimental tests is to find characteristic curves suitable for the design in specific functioning conditions. Some of the tests results are described in Fig. 10; these curves are referred to a prototype composed by oscillating magnet on magnetic suspension, 4 coils connected in parallel and current rectifier. The resonance frequency of the harvester is $3.2 \mathrm{~Hz}$ and the tests are conducted at $0.12 \mathrm{~g}$ acceleration.

This particular typology of inductive generator has been addressed to applications in railway bogies [7], axels of industrial vehicles [9], telescopic arms, operative machines and sport equipment [8], where the excitation frequency range is localized at low values.

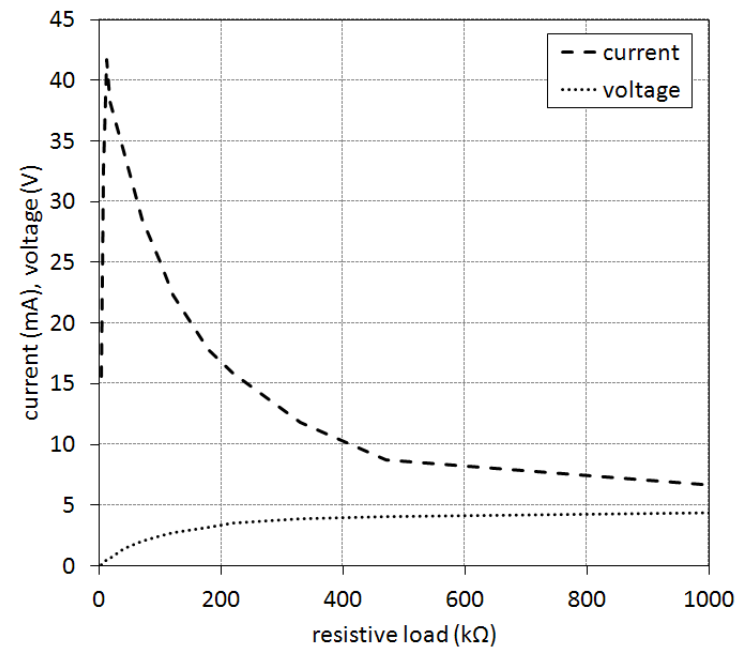

(a)

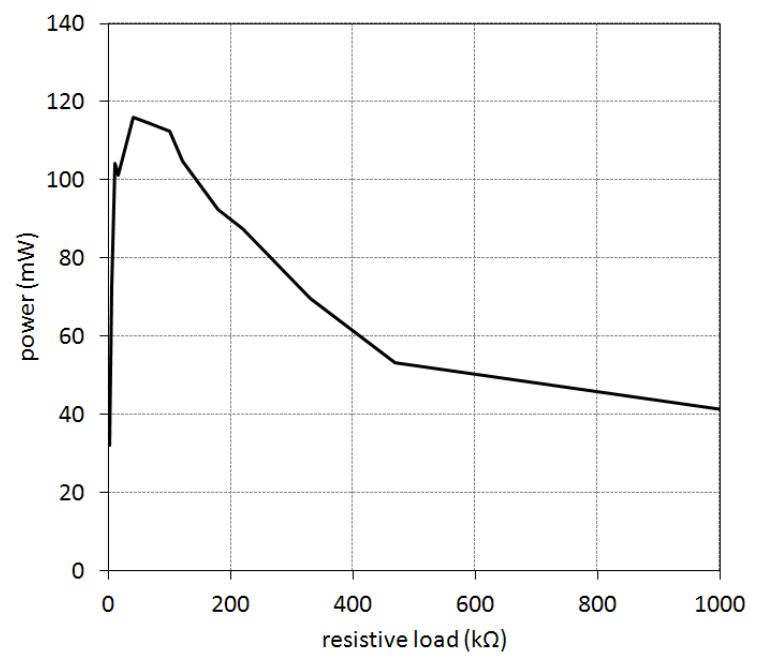

(b)

Figure 10: Electric output of the magnetic inductive generator with 4 coils (3.2 Hz, $0.12 \mathrm{~g}$ ): (a) current and voltage and (b) output power. 


\section{CONCLUSIONS}

his work introduced the most important steps of the design autonomous systems for converting the kinetic energy of mechanical vibrations in electric power. The activity is focused on the self-powered sensing system design, instead of traditional simpler energy harvester design, with the aim of responding more exhaustively to the requirements of the application. From the energetic balance viewpoint, the concept of duty cycle is crucial, as well as the analysis of input vibration spectra through its PSD and its time-dependent variability. These aspects, together with bandwidth amplification strategies and resonance tuning, provide the required information for selecting the proper transducer typology and for defining its dimension and dynamic properties.

\section{REFERENCES}

[1] S. Roundy, J. of Intelligent Materials and Structures, 16 (2005) 809.

[2] S. Roundy, P.K. Wright, J. Rabaje, Computer Communications, 26 (2003) 1131.

[3] S. Priya, D.J. Inman, "Energy harvesting technologies", New York, Springer (2008).

[4] G. De Pasquale, A. Somà, F. Fraccarollo, In: Proceedings of the Institution of Mechanical Engineers - Part C: Journal of Mechanical Engineering Science, 226 (2011).

[5] G. De Pasquale, A. Somà, N. Zampieri, J. of Computational and Nonlinear Dynamics, 7 (2012) 041011.

[6] G. De Pasquale, C. Siyambalapitiya, A. Somà, J. Wang, In: Proc. International Conference on Electromagnetics in Advanced Applications (ICEAA), Torino 465-468, (2009).

[7] A. Somà, G. De Pasquale, Device for diagnosing railway bogies by applying an energy-autonomous measuring and transmitting bolt, and corresponding control method, PCT Patent n. WO2011/117718 (2011).

[8] A. Somà, G. De Pasquale, F. Fraccarollo, Racchetta da sci o trekking dotata di dispositivo harvester magneticoinduttivo di generazione elettrica, Patent n. TO2011A000844 (2011).

[9] A. Somà , G. De Pasquale, Sistema autoalimentato mediante harvester per monitoraggio ed infomobilità di applicazioni veicolistiche multinodali wireless, Patent n. TO2011A000694 (2011).

[10] R. Elfrink, T.M. Kamel, M. Goedbloed, S. Matova, D. Hohlfeld, Y. van Andel, R. van Schaijk, J. of Micromechanics and Microengineering, 19, 094005 (2009).

[11] S. Cheng, D.P. Arnold, J. of Micromechanics and Microengineering, 20 (2010) 025015.

[12] Y. Chiu, V.F. Tseng, J. of Micromechanics and Microengineering, 18 (2008) 104004.

[13] Y. Suzuki, D. Miki, M. Edamoto, J. of Micromechanics and Microengineering, 20 (2010) 104002.

[14] http://www.cedrat-technologies.com. 